\title{
Infrared Spectroscopy of the Tropyl Radical in Helium Droplets
}

\author{
Matin Kaufmann, ${ }^{\dagger}$ Daniel Leicht, ${ }^{\dagger}$ Martina Havenith, ${ }^{\dagger}$ Bernadette M. \\ Broderick, $\stackrel{\ddagger}{\ddagger}$ and Gary E. Douberly ${ }^{*, \ddagger}$ \\ $\dagger$ Department of Physical Chemistry II, Ruhr-University Bochum, 44801 Bochum, Germany \\ $\ddagger$ Department of Chemistry, University of Georgia, Athens, GA 30602-2556, United States \\ E-mail: douberly@uga.edu
}




\section{Supporting Information}

\section{Spectroscopic Model}

\section{Energy Levels}

We calculate the effects of the vibronic coupling following the approach outlined in ${ }^{1}$, that has been used for the considerations in $^{2}$ and $^{3}$. We start with the Hamiltonian

$$
\hat{H}=\hat{H}_{T}+\hat{V}
$$

where $\hat{H}_{T}$ is the kinetic energy operator and $\hat{V}$ the effective potential including the JahnTeller coupling as off-diagonal elements, both in terms of the vibrational normal modes $Q_{i}$. We neglect both, the rotational as well as the spin-orbit interaction, since the rotational constants for tropyl are $\sim 0.1 \mathrm{~cm}^{-1}$ and the spin-orbit coupling has been described to be negligible. $^{2,3}$

For the potential $\hat{V}$ only the terms up to second polynomial order in the normal modes are taken into account, with the non-zero contributions

$$
\begin{aligned}
\hat{H}_{H O} & =\sum_{i=1}^{p} \sum_{r=+,-} \frac{1}{2} \lambda_{i}\left|Q_{i, r}\right|^{2} \\
\hat{H}_{\text {lin }} & =\sum_{i=1}^{p} \sum_{r=+,-} k_{i} Q_{i, r} \\
\hat{H}_{\text {quad }} & =\sum_{i=1}^{p} \sum_{r=+,-} \frac{1}{2} g_{i i}\left(Q_{i, r}\right)^{2}
\end{aligned}
$$

with $\hat{H}_{H O}$ the harmonic oscillator term, $\hat{H}_{\text {lin }}$ the linear and $\hat{H}_{\text {quad }}$ the quadratic Jahn-Teller term for the $p$ Jahn-Teller active modes $Q_{i, r}$ and the index $r$ summing over the two electronic states $|\Lambda\rangle$ that are degenerate at the symmetric configuration. The parameters are defined 


$$
\begin{aligned}
\lambda_{i} & =\left\langle\Lambda\left|\frac{\partial^{2} \hat{V}}{\partial Q_{i,+} \partial Q_{i,-}}\right| \Lambda\right\rangle \\
k_{i} & =\left\langle\Lambda\left|\frac{\partial \hat{V}}{\partial Q_{i, r}}\right|-\Lambda\right\rangle \\
g_{i i} & =\left\langle\Lambda\left|\frac{\partial^{2} \hat{V}}{\left(\partial Q_{i, r}\right)^{2}}\right|-\Lambda\right\rangle,
\end{aligned}
$$

with the force constant $\lambda_{i}$, the linear coupling constant $k_{i}$ and the quadratic coupling constant $g_{i i}$ for the $i^{\text {th }}$ Jahn-Teller active mode.

Additional terms, like the cross-quadratic and the bilinear coupling terms are neglected, as they are assumed to be weak in accordance to ${ }^{1}$. No coupling between distinct modes is expected, therefore all $p$ Jahn-Teller modes can be treated separately and the index $i$ may be dropped. Additionally, the harmonic oscillator energy $\omega$ and unitless parameters $D$ for the linear and $K$ for the quadratic coupling are used

$$
\begin{aligned}
\omega & =\frac{1}{2 \pi c} \sqrt{\frac{\lambda}{\mu}} \\
D & =\frac{k^{2}}{2 \hbar} \sqrt{\frac{\mu}{\lambda^{3}}} \\
K & =\frac{g}{\lambda},
\end{aligned}
$$

with the reduced mass $\mu$, the force constant $\lambda$ and the coupling constants $k$ and $g$ for a distinct mode.

As the basisfunctions for the vibronic wavefunctions, the product of the electronic eigenfunctions and the harmonic oscillator eigenfunctions is chosen

$$
\begin{aligned}
\mid \text { electronic }\rangle & =|\Lambda\rangle, \quad \Lambda= \pm 1 \\
\mid \text { vibrational }\rangle=|v, l\rangle, & v=0,1,2, \ldots \\
& l=-v,-v+2, \ldots, v-2, v,
\end{aligned}
$$


with $\Lambda$ the quantum number for the two degenerate electronic states, $v$ the vibrational excitation of the degenerate mode and $l$ the vibrational angular momentum.

Since the Hamiltonian no longer contains coupling between different vibrational modes, the eigenvalue problem can be solved separatly for each vibrational mode. The Integrals $\left\langle l, v\left|\left\langle\Lambda\left|\hat{H}_{T}+\hat{H}_{H O}\right| \Lambda^{\prime}\right\rangle\right| v^{\prime}, l^{\prime}\right\rangle,\left\langle l, v\left|\left\langle\Lambda\left|\hat{H}_{l i n}\right| \Lambda^{\prime}\right\rangle\right| v^{\prime}, l^{\prime}\right\rangle$ and $\left\langle l, v\left|\left\langle\Lambda\left|\hat{H}_{\text {quad }}\right| \Lambda^{\prime}\right\rangle\right| v^{\prime}, l^{\prime}\right\rangle$ are evaluated and the Hamiltonian diagonalized to obtain the energies and wavefunctions of the vibronic states.

The matrix elements follow as

$$
\begin{aligned}
& \left\langle l, v\left|\left\langle \pm\left|\hat{H}_{T}+\hat{H}_{H O}\right| \pm\right\rangle\right| v, l\right\rangle=\omega(v+1) \\
& \left\langle l, v\left|\left\langle \pm\left|\hat{H}_{l i n}\right| \mp\right\rangle\right| v^{\prime}, l^{\prime}\right\rangle=\omega\left[\sqrt{D\left(v \mp(-1)^{s_{1}} l+2\right)} \times \delta_{v+1, v^{\prime}} \delta_{l \mp(-1)^{s_{1}, l^{\prime}}}\right. \\
& \left.+\sqrt{D\left(v \pm(-1)^{\left.s_{1} l\right)}\right.} \times \delta_{v-1, v^{\prime}} \delta_{l \mp(-1)^{s_{1}, l^{\prime}}}\right] \\
& \left\langle l, v\left|\left\langle \pm\left|\hat{H}_{\text {quad }}\right| \mp\right\rangle\right| v^{\prime}, l^{\prime}\right\rangle= \\
& \frac{\omega K}{4}\left[\sqrt{\left(v \mp(-1)^{s_{2}} l\right)\left(v \mp(-1)^{s_{2}} l-2\right)} \times \delta_{v-2, v^{\prime}} \delta_{l \pm 2(-1)^{s_{2}, l^{\prime}}}\right. \\
& +2 \sqrt{\left(v \pm(-1)^{s_{2}} l+2\right)\left(v \mp(-1)^{s_{2}} l\right)} \times \delta_{v, v^{\prime}} \delta_{l \pm 2(-1)^{s_{2}, l^{\prime}}} \\
& \left.+\sqrt{\left(v \pm(-1)^{s_{2}} l+4\right)\left(v \pm(-1)^{s_{2}} l+2\right)} \times \delta_{v+2, v^{\prime}} \delta_{l \pm 2(-1)^{s_{2}, l^{\prime}}}\right]
\end{aligned}
$$

with symmetry dependent parameters $s_{k}=0,1$ (see Eqn. (26) in Ref. ${ }^{1}$ ). In the case of tropyl, $s_{1}=0$ for the $E_{3}^{\prime}$ mode and $s_{2}=1$ for the $E_{2}^{\prime}$ mode.

\section{Infrared Selection Rules}

To estimate the dipole transition probabilities, the integrals of the form $\left\langle\Psi^{\prime}|\hat{\mu}| \Psi^{\prime \prime}\right\rangle$ are evaluated. This has been done for the linear Jahn-Teller coupling in ${ }^{4}$. States with different $l$ quantum numbers are coupled by the Jahn-Teller effect, therefore the energy eigenfunctions are expressed in terms of the quantum numbers $\Lambda, v$ and $j$, with $j=l+(-1)^{s_{1}} \frac{1}{2} \Lambda$. 
The matrix elements for the vibrational transitions have the general form

$$
\left[\left\langle j^{\prime}, v^{\prime},+\right|\left\langle j^{\prime}, v^{\prime},-\right|\right]\left[\begin{array}{cc}
\langle+|\hat{\mu}|+\rangle & \langle+|\hat{\mu}|-\rangle \\
\langle-|\hat{\mu}|+\rangle & \langle-|\hat{\mu}|-\rangle
\end{array}\right]\left[\begin{array}{l}
\left|+, v^{\prime \prime}, j^{\prime \prime}\right\rangle \\
\left|-, v^{\prime \prime}, j^{\prime \prime}\right\rangle
\end{array}\right]
$$

with $\hat{\mu}$ the dipole moment operator. When expanding the matrix elements up to first order in the normal coordinates, Eqn. (7) can be broken down into primitive expressions. Evaluating these yields coefficients that vanish completely except for specific changes in the quantum numbers. By allowing all non-zero contributions, the following selection rules have been determined by ${ }^{4}$ :

$$
\Delta \Lambda= \pm 2, \quad \Delta v= \pm 1, \quad \Delta j= \pm 1, \pm 2
$$

\section{References}

(1) Barckholtz, T. A.; Miller, T. A. Quantitative insights about molecules exhibiting JahnTeller and related effects. Int. Rev. Phys. Chem. 1998, 17, 435-524.

(2) Stakhursky, V. L.; Sioutis, I.; Tarczay, G.; Miller, T. A. Computational investigation of the Jahn-Teller effect in the ground and excited electronic states of the tropyl radical. Part I. Theoretical calculation of spectroscopically observable parameters. J. Chem. Phys. 2008, 128, 084310.

(3) Sioutis, I.; Stakhursky, V. L.; Tarczay, G.; Miller, T. A. Experimental investigation of the Jahn-Teller effect in the ground and excited electronic states of the tropyl radical. Part II. Vibrational analysis of the $\tilde{A}^{2} E_{3}^{\prime \prime}-\tilde{X}^{2} E_{2}^{\prime \prime}$ electronic transition. J. Chem. Phys. 2008, 128, 084311.

(4) Child, M. S.; Longuet-Higgins, H. C. Studies of the Jahn-Teller Effect III. The Rotational and Vibrational Spectra of Symmetric-top Molecules in Electronically Degenerate States. Philos. T. Roy. Soc. A 1961, 254, 259-294. 